\title{
Diabetic nephropathy: where do we stand
}

\begin{abstract}
Huge global burden of Diabetes with increasing trend in low and middle income group countries has impact on prevalence of diabetic kidney disease which has been found to be commonest cause of CKD. The care of CKD is costly and its socio-economic impact would be tremendous. Physicians often miss to look at socio-economical aspect in the zeal of doing more good while managing the diabetic patient. With more number of treatment novelties at hand, most of which are not cheaper, a cautious and conscious consideration towards socio-economical aspect with aim to keep management cost of the problem manageable is required very much.
\end{abstract}

Keywords: diabetes mellitus, diabetic nephropathy, diabetic kidney disease, chronic kidney disease, albuminuria, sglt inhibitors
Volume 4 Issue 2 - 2017

\author{
Chandra Mauli Jha,' Dastoor $\mathrm{H}^{2}{ }^{2}$ Hatem \\ Ebeid $^{3}$ \\ 'HOD Department of Nephrology and Dialysis services, GDC \\ Hospital, Abu Dhabi, UAE \\ ${ }^{2}$ HOD Department of Nephrology and Dialysis Services, Rahba \\ Hospital, Abu Dhabi, UAE \\ ${ }^{3}$ Nephrologist, Kairo Kidney Centre, Egypt
}

Correspondence: Chandra Mauli Jha, HOD Department of Nephrology and Dialysis services, GDC Hospital,Abu Dhabi, UAE, Post Box 6I358, Abu Dhabi, UAE, Tel $97 \mid 501096$ 345, Fax 97I 25580482,Email chmjha@gmail.com

Received: February 26, 2017 | Published: March 2, 2017
Abbreviations: DM, Diabetes Mellitus; DKD, Diabetic Kidney Disease; CKD, Chronic Kidney Disease; RAAS, Renin Angiotensin Aldosterone system; SGLT, Selective Sodium-glucose Transporter; e-GFR, Estimated Glomerular Filtration Rate; UACR, Urinary Albumin Creatinine Ratio, ESRD, End Stage Renal Disease; MI, Myocardial Infarction; ACEi, Angiotensin Converting Enzyme Inhibitors; ARB, Angiotensin Receptor Blocker

\section{Introduction}

The diabetes mellitus has emerged as an important component of global burden of noncommunicable diseases. It is associated with significant morbidity and mortality. The incidence and prevalence is rising in developing countries. Approximately 25 to $40 \%$ of diabetics develop renal complications and it remains the largest contributor to ESRD population in many countries. The chronicity and complications brings huge personal \& societal economic burden. The economical aspect of management remains the blind spot. Here we shall attempt to explore why there is a need to change our focus towards the economical aspect of plan and enforcement of management of diabetes and diabetic kidney disease.

\section{Discussion}

Diabetes mellitus has emerged as the global health problem of enormous magnitude due to its high prevalence and its complications - macrovascular and microvascular - affecting the eyes, heart, circulation and the kidneys which lead to disability and premature death in the affected population. As per the latest WHO Estimate the word wide prevalence of diabetes in 2014 in population older than 18 years is about $8.5 \%{ }^{1}$ The disease no longer holds the status of the disease of the rich. It prevalence has persistently risen during last three decades in countries of all socioeconomic group. Increase in Prevalence in 2014 compared to that in 1980 is highest in the Middle
East region (5.9\% in 1980 and $13.7 \%$ in 2014) followed by African, South-East Asian and Western Pacific Region. ${ }^{1}$ A pooled analysis of 751 population-based studies with $4 \cdot 4$ million participants estimates that forty per cent of this increase is estimated to result from population growth and ageing, $28 \%$ from a rise in age-specific prevalence, and $32 \%$ from the interaction of the two. ${ }^{2}$

Type 2 diabetes mellitus constitutes majority of the diabetic population. Up to 20 to $40 \%$ of these diabetic patients do develop diabetic nephropathy. ${ }^{3}$ We have shifted from an earlier view of type 2 diabetes mellitus as a relatively benign condition compared to type 1 diabetes mellitus without much effect on life expectancy and renal function, especially in elderly [4] to that that it is not so and the risk of kidney disease is similar in the two types of diabetics. ${ }^{5}$ Prevalence of DKD in the united States in 2008 compared to that in 1988 has increased in proportion to the rise in the prevalence of the diabetes. ${ }^{6}$ Approximately four to five times larger number of type 2 diabetics than type 1 contributes largely to epidemic of chronic Kidney disease including ESRD. ${ }^{6}$ Diabetes remains leading cause of end-stage renal disease (ESRD) in USA, approximately $50 \%$ cases, with a trend stabilized over the past few years. ${ }^{7}$ Though, this stabilization appears as a positive note, the finding that prevalence of DKD had not decreased despite increased use of glucose-lowering medications and RAAS inhibitors, may indicate not to be so. ${ }^{6}$ The situation of stable prevalence in USA is in contrast to that in the developing countries where the majority of world diabetic population resides and where the diabetic prevalence has increasing trend. ${ }^{1,3}$

Diabetes is preventable ${ }^{8}$ and so is the diabetic kidney disease. The later can be achieved by prevention of diabetes and its good care in those who have developed the disease. Early diagnosis of DKD and its improved care would have effect on the number of DKD patients progressing towards ESRD. UNO has held two meetings of heads of state on a health-related issue-on HIV in 2001 and on 
noncommunicable diseases in 2011. The later has set a target of " 25 by 25 " which aims, jointly endorsed by UNO and WHO, for a $25 \%$ reduction by 2025 in mortality from noncommunicable diseases among persons between 30 and 70 years of age, in comparison with mortality in 2010. Diabetes and associated diabetic kidney disease being part of the group of noncommunicable diseases remained a target of the convention.

The magnitude of the problem of the diabetes and diabetic kidney disease has two arenas, first due to associated cardiovascular morbidity and mortality and second due to huge socio-economic aspect, nevertheless the two being interconnected in some ways. While the physicians are always concerned with the former part of problem, they often miss to give importance to the later part of the problem. The disease causes economic loss to person with diabetes and their families. The treatment cost and the loss of work affects the health system and national economy. Cost of medicine and investigations are important parameters which must be optimized to deliver the best. An impulse to prescribe newer costlier treatment without robust evidence to benefit significantly must be fought by the caring physicians. For example, WHO global report mentions increasing trend of costlier analogue Insulin being prescribed without any little evidence that it had significant advantages over cheaper human insulins. ${ }^{11}$ It has been noted that evidence-based therapies like use of low cost generic antihypertensive agents and statins were prescribed to lesser number of patients in the low and middle-income countries. ${ }^{10}$

There is need for the development of evidence based guidelines addressing the issue, if a costlier therapy is certainly better than the other less costlier or not, and how much if so. The importance of such consideration becomes evident when we look at the discrepancy in the national expenditure for diabetic care to that of diabetic population in the countries of different economical status. China, India \& USA have highest number of diabetic patients -109 million, 69 million and 29 million respectively. Among the top ten countries spending maximum for diabetic care, USA remains at the top by spending an amount of 320 billion dollars annually against 51 billion dollars annually by China. While India not being in the club of top ten countries. ${ }^{3} A n$ unaffordable treatment is of no benefit at all no matter whatever great benefit it may have! Hereupon we shall focus to areas of diabetic kidney disease which is more likely to be affected by costlier novel therapies or which has implication on its management being costlier.

To begin with, there is great need to take preventive measures to halt progression of diabetic epidemic and DKD. Early recognition of diabetic kidney disease with associated risk factors for progression followed by preventive measures against progression should be part of development of national planning and every physician should aim to be part of that. Behavioral pattern leading to use of tobacco, alcohol, physical inactivity and poor diet and some genetic influence determines community risk of high blood pressure, hypercholesterolemia, obesity, glucose intolerance and diabetes. ${ }^{11}$ It brings need of education and development of social environment promoting and encouraging healthy life style choices in order to curb the number of DKD. The physicians and health services are required to be focused on early detection and cost-effective management of noncommunicable diseases and their risk factors. Social, educational and legislative measures to reduce intake of sugar, salt, alcohol, saturated fat, smoking and promoting physical activity should be the aim of family, society and government and physicians must push for that. Developments in Information and communication technology such as mass-media messaging and use of social platforms can enable health care providers to play an effective role in promoting awareness leading to prevention, improving diagnosis and management. ${ }^{12}$ Kidney care need planning in that direction in low and middle income group countries is dealt in an extensive meeting report article which could guide in tailoring the requirement of different population. ${ }^{13}$

It has been noted that though the absolute risks of ESRD, all-cause mortality, and cardiovascular mortality were higher in patients with chronic kidney disease and diabetes than in those without diabetes throughout the ranges of eGFR and albuminuria, the relative risks of these outcomes were the same. ${ }^{13}$ So, clinically the diabetic kidney disease detected by the assessment of eGFR and UACR remains the tools for CKD patients whether with or without diabetes. ${ }^{14,15}$ Both of these tools have limitations and there is need of search of better identification methods, especially for people at high risk of DKD. ${ }^{16}$ Next step remains cardiovascular risk assessment in indentified DKD patients since cardiovascular death remains the major cause of death among them. Cardiovascular risk Prevention through targeting various risk factors like control of high blood pressure, dyslipidemia, cessation of tobacco exposure etc. takes a central state after identifying the CKD. Yet, it must be borne in mind that the risk factors unique to the DKD population to improve risk stratification as well as treatment strategies are not known in depth and research towards this direction is required. Safety has to be ensured all along while aiming for efficacy.

Regarding control of high blood pressure as a cardiovascular risk factor, present recommendations of blood pressure control to target $\leq 140 / 90 \mathrm{~mm} \mathrm{Hg}$ by Joint National Committee (JNC) 8 and KDIGO to reduce CVD mortality and slow CKD progression is based on outcome in randomized trials of non-DKD participant. "KEEP" study showed that lower blood pressure in CKD stage 3 and later is associated with higher incident rates of ESRD. ${ }^{17}$ These guidelines recommend ACEi as drug of choice for control of high blood pressure among diabetics and patients of CK but there remains need of evaluation of ACEi \& $\mathrm{ARB}$ in advanced stage of CKD over its benefit versus progression to ESRD due to ischemic effect. Earlier double RAAS blockade has been abandoned after ON-TARGET Trial. ${ }^{18}$ Every effort must be made to slow progression of diabetic CKD to ESRD which increases social \& medical cost several folds. Similarly all potential nephrotoxic drugs should be hunted and limited in prescribing habit. The proton pumps inhibitors which occupy a place among "common drugs prescribed chronically" among diabetics have been found to increase risk of CKD progression. ${ }^{19}$ Physicians must aim to prevent any kind of acute kidney injury since it has emerged as other biomarkers of future development of CKD. ${ }^{20}$

The importance of good glycemic control is established beyond doubt in type 1 and type 2 diabetics in DCCT and UKPDS studies respectively. ${ }^{21,22}$ UKPDS also showed that type of treatment for glycemic control matters less. ${ }^{22}$ At the same time ACCORD study cautioned us about unknown risk of intensive glycemic control and made us to raise our target $\mathrm{HbA1C}$ values. ${ }^{23}$ Regular monitoring of eGFR and modification of drug dose (such as Metformin) is recommended. By the time, CKD patients reach to stage 4 to 5 , the glycemic control needs to be less aggressive. There is no hard data about the optimal glycemic target in advanced CKD patients. Some recent data is in favour of higher HbA1C target from 6 or 7 to $9 \%$ which is associated with better survival rates in both haemodialysis ${ }^{24,25}$ and peritoneal dialysis patients. ${ }^{26,27}$ Recent novel therapies added to 
control hyperglycemia belongs to three broad groups of compounds, glucagon-like peptide-1 (GLP-1) agonists, which stimulate insulin release; dipeptidyl peptidase-4 (DPP-4) inhibitors, which act along the same pathway and prevent the breakdown of GLP-1, also stimulating insulin release; and, sodium glucose co-transporter (SGLT) inhibitors, largely inhibitors of type 2 SGLT, which prevent the resorption of glucose by the proximal tubule.

These drugs are costly and likely to be prescribed more and more in the future. Efficacy and benefit of these drugs are needed to be evaluated carefully against commercial pressure and desire to use a drug novel, especially when the findings of several trials of these drugs are not uniformly in one direction. DPP-4 inhibitors Alogliptin, Saxagliptin, Sitagliptin and GLP-1 agonist lixisenatide evaluated in "Examine" trial, ${ }^{28}$ "SAVOR-TIMI 53" trial, ${ }^{29}$ TECOS Trial $^{30}$ and ELIXA Trial ${ }^{31}$ respectively did not show any decrease in cardiovascular outcome when compared to the control of diabetes with the use of other methods. More recently Liraglutide, a GLP-1 agonist, in LEADER Trial, ${ }^{32}$ showed lower rate of cardiovascular death, lower all cause mortality, lesser rate of development of nephropathy (albuminuria) compared to placebo though the incidence of MI, stroke $\&$ hospitalization were same and there was higher rate of statistically non-significant retinopathy.

SGLT 2 inhibitor Empagliflozin in EMPA-REG OUTCOME trial $^{33}$ showed lower cardiovascular mortality, slower progression of kidney disease and lower rates of clinically relevant renal events compared to placebo, though there was no decrease in rate of MI, stroke or hospitalization. Result of EMPA-REG OUTCOME trial does not match with results of studies on other SGLT 2 inhibitors. Why "EMPA-REG OUTCOME" trial \& "LEADER" trial results are at variance from other trials on drugs of these groups requires to be searched further. We require well deigned trials uniformly comparing the cheaper medicines against novel drugs to clearly define the treatment for mass and the place of costlier drugs for that. That would also help in industrial and legislative measures to make it work in the entire design. In conclusion, we have more unknown areas than known in the management of DKD patients and till we explore those well, we are required to tread the path more cautiously.

\section{Acknowledgement}

None.

\section{Conflicts of interest}

The author declares there is no conflict of interest.

\section{References}

1. Global report on diabetes. World Health Organization ISBN 978924 1565257 (NLM classification: WK 810). 2016.

2. NCD Risk Factor Collaboration (NCD-RisC). Worldwide trends in diabetes since 1980: a pooled analysis of 751 population-based studies with 4.4 million participants. Lancet. 2016;387(10027):1513-1530.

3. 2015 IDF Diabetes Atlas, (7th edn). 2015.

4. Fabre J, Balant LP, Dayer PG, et al. The kidney in maturity onset diabetes mellitus: a clinical study of 510 patients. Kidney Int. 1982;21(5):730-738.

5. Hasslacher C, Ritz E, Wahl P, et al. Similar risks of nephropathy in patients with type I or type II diabetes mellitus. Nephrol Dial Transplant. 1989;4(10):859-863.
6. de Boer IH, Rue TC, Hall YN, et al. Temporal trends in the prevalence of diabetic kidney disease in the United States. JAMA. 2011;305(24):25322539 .

7. Chapter 1: Incidence, Prevalence, Patient Characteristics, and Treatment Modalities, 28th Annual Data Report of the United States Renal Data System (USRDS). 2016.

8. Diabetes Prevention Program (DPP). 2002.

9. 2015 Noncommunicable diseases. Geneva: World Health Organization.

10. Ezzati M, Riboli E. Can noncommunicable diseases be prevented? Lessons from studies of populations and individuals. Science. 2012;337(6101):1482-1487.

11. Ezzati M, Riboli E. Behavioral and dietary risk factors for noncommunicable diseases. N Engl J Me. 2013;369:954-964.

12. Hunter DJ, Reddy KS. Noncommunicable Diseases. $N$ Engl J Med. 2013;369:1336-1343.

13. V Jha. Kidney care in low- and middle-income countries; Kidney International. 2016;90:1164-1174.

14. Fox CS, Matsushita K, Woodward M, et al. Chronic Kidney Disease Prognosis Consortium. Associations of kidney disease measures with mortality and end-stage renal disease in individuals with and without diabetes: a meta-analysis. Lancet. 2012;380(9854):1662-1673.

15. KDIGO. Chapter 2: definition, identification, and prediction of CKD progression. Kidney Int. 2013; Suppl (2011)3:63-72.

16. Tuttle KR, Bakris GL, Bilous RW, et al. Diabetic Kidney Disease: A Report From an ADA Consensus Conference. Diabetes Care. 2016;37(10):2864 2883.

17. Peralta CA, Norris KC, Li S, et al. Blood pressure components and endstage renal disease in persons with chronic kidney disease: the Kidney Early Evaluation Program (KEEP). Arch Intern Med. 2012;172(1):41-47.

18. ONTARGET Investigators, Yusuf S, Teo KK, et al. Telmisartan, Ramipril, or Both in Patients at High Risk for Vascular Events. $N$ Engl J Med. 2008;358(15):1547-1559.

19. Xie Y, Bowe B, Li T, et al. Proton Pump Inhibitors and Risk of Incident CKD and Progression to ESRD. J Am Soc Nephrol. 2016;27(10):31533163 .

20. Monseu M, Gand E, Saulnier PJ, et al. Acute Kidney Injury Predicts Major Adverse Outcomes in Diabetes: Synergic Impact With Low Glomerular Filtration Rate and Albuminuria. Diabetes Care. 2015;38(12):2333-2340.

21. Nathan DM, Cleary PA, Backlund JY, et al. Intensive Diabetes Treatment and Cardiovascular Disease in Patients with Type 1 Diabetes. $N$ Engl J Med. 2005;353(25):2643-2653.

22. UK Prospective Diabetes Study (UKPDS) Group. Intensive blood-glucose control with sulphonylureas or insulin compared with conventional treatment and risk of complications in patients with type 2 diabetes (UKPDS 33). Lancet. 1998;352(9131):837-853.

23. Action to Control Cardiovascular Risk in Diabetes Study Group, Gerstein $\mathrm{HC}$, Miller ME, et al. Effects of Intensive Glucose Lowering in Type 2 Diabetes. N Engl J Med. 2008;358(24):2545-2559.

24. Ricks J, Molnar MZ, Kovesdy CP, et al. Glycemic control and cardiovascular mortality in hemodialysis patients with diabetes: a 6-year cohort study. Diabetes. 2012;61(3):708-715.

25. Kalantar-Zadeh K, Kopple JD, Regidor DL, et al. A1C and survival in maintenance hemodialysis patients. Diabetes Care. 2007;30(5):1049 1055 . 
26. Hoshino J, Mehrotra R, Rhee CM, et al. Using hemoglobin A1c to derive mean blood glucose in peritoneal dialysis patients. Am J Nephrol. 2013;37(5):413-420.

27. Duong U, Mehrotra R, Molnar MZ, et al. Glycemic control and survival in peritoneal dialysis patients with diabetes mellitus. Clin J Am Soc Nephrol. 2011;6(5):1041-1048.

28. White WB, Cannon CP, Heller SR, et al. Alogliptin after acute coronary syndrome in patients with type 2 diabetes. N Engl J Med. 2013;369:13271335 .

29. Scirica BM, Bhatt DL, Braunwald E, et al. Saxagliptin and cardiovascular outcomes in patients with type 2 diabetes mellitus. $N$ Engl $\mathrm{J} \mathrm{Med}$. 2013;369(14):1317-1326
30. Green JB, Bethel MA, Armstrong PW, et al. Effect of sitagliptin on cardiovascular outcomes in type 2 diabetes. $N$ Engl $\mathrm{J}$ Med. 2015;373(3):232-242

31. Pfeffer MA, Claggett B, Diaz R, et al. Lixisenatide in patients with type 2 diabetes and acute coronary syndrome. NEngl J Med. 2015;373(23):22472257.

32. Marso SP, Daniels GH, Brown-Frandsen K, et al. Liraglutide and cardiovascular outcomes in type 2 diabetes. $N$ Engl $\mathrm{J} \mathrm{Med}$. 2016;375(4):311-322.

33. Zinman B, Wanner C, Lachin JM, et al. Empagliflozin, cardiovascular outcomes, and mortality in type 2 diabetes. $N$ Engl J Med. $2015 ; 373(22): 2117-2128$ 